\title{
Binder-Free Monoliths of Graphene Dried Foam Films Covering Polyaniline for Supercapacitors Electrode
}

\author{
Wufeng Chen, ${ }^{a, b}$ Junhao Chen, ${ }^{a}$ and Lifeng Yan ${ }^{\star, a}$ \\ ${ }^{a}$ Department of Chemical Physics, iCHEM, University of Science and Technology of China, \\ Hefei, Anhui 230026, China \\ ${ }^{b}$ Ashine Advanced Carbon Material (Changzhou) Co., Ltd., Changzhou, Jiangsu, China \\ Email: Ifyan@ustc.edu.cn (L. Y.)
}

\begin{abstract}
Dried foam films (DFF) of graphene oxide (GO) has been prepared by dipping a titanium mesh in the solution of GO, and then it was reduced by electrochemical method. Next, the as-prepared water-containing reduced grapheme oxide (rGO) DFF film works as electrode for electrocpolymerization of aniline onto its surface for preparing a polyaniline/graphene monolith while the titanium mesh acts as the electron collector. The as-obtained composite film is binder-free and has highly surface area, conductivity, and can be used for supercapacitor electrode. Then network of plenty polyaniline nanoparticles formed on the surface of rGO film by electrochemical polymerization, which works as the material for pseudocapacitance. When the film is used as a supercapacitor electrode, the maximum specific capacitance is as high as $633.4 \mathrm{~F} \mathrm{~g}^{-1}$ and the specific capacitance retains $75 \%$ of the initial after constant charge discharge 5000 cycles at current density of $10 \mathrm{~A} \mathrm{~g}^{-1}$, indicating that the nanocomposite is a suitable active material for supercapacitors.
\end{abstract}

Keywords graphene, polyaniline, dried foam film, supercapacitors, electrode

\section{Introduction}

As a kind of energy storage device with high power density and long lifespan, supercapacitors have attracted great interest owing to their high power density, excellent long cycling life and fast charging/discharging rate. ${ }^{[1]} \mathrm{New}$ nanostructured electrode materials should be developed to improve the specific capacitance and increase the operating voltage, and the most widely used materials for electrodes of supercapacitors are carbon based materials with high surface area, such as carbon fibers, carbon nanotube, etc. ${ }^{[2]}$ Graphene has been paid much attention since the first preparation of in 2004, ${ }^{[3]}$ and it has showed great potential application in supercapacitors due to its highly specific area and conductivity. ${ }^{[4]}$ However, electrodes based on graphene powder is usually required to use a binder or additives to stick the active materials onto the surface of collector, but their presence would decrease the conductivity of the electrode and result in poor performance of the supercapacitors, ${ }^{[5]}$ and monolith electrodes are expected. ${ }^{[6]}$ Recently, free-standing thin film supercapacitors have been attracted much attention, because they can be directly used as working electrodes in free of binders or additives. ${ }^{[7]}$ It is well known that graphene oxide (GO) can be well dispersed in aqueous solution and easily processed than graphene, which is difficult to disperse in solvents at high concentration. Thus, it is better to process GO to free-standing film or bulk materials following reduction for developing new kinds of monolith graphene materials as electrode. Electrochemical reduction of graphene oxide (GO) is an effective method to prepare reduction graphene ( $\mathrm{rGO}$ ) of low defect and high capacitance. ${ }^{[8]}$ Recently, our group had developed a method to obtain a fantasy free-standing dried GO foam film, which can be reduced to an ultrathin graphene film. ${ }^{[9]}$ It may be used to prepare binder-free monoliths of graphene or its composite for supercapacitors electrodes. Here, the frame of titanium mashes was used as both the support of free-standing micro-films and collector electrode, and the dried foam films (DFF) of GO was obtained at first by this method. Then, it was reduced to latticed rGO DFF film, which works directly as the active materials for the electrodes of supercapacitor. Compared with traditional carbon based double-layer capacitors, pseudo-capactive electrode materials could increase electrochemical capacitances and energy densities. The most selective materials are metal oxides. In addition, conductive polymers has been paid much attention for their excellent performance for supercapacitors, such as polypyrrole, polyaniline, etc. ${ }^{[10]}$

Herein, rGO DFF films has been prepared at first by the dried foam film method following electrochemical reduction, and then aniline monomer was electropolymerized on the surface of rGO DFF. A binder-free monolith of polyaniline/rGO (PANI/rGO) DFF films was obtained as supercapacitor electrodes, and the performance has been studied.

\section{Experimental}

\section{Materials}

Graphite powder, natural briquetting grade, 8000 meshes, $99.95 \%$ (metals basis), and aniline $(99.5 \%)$ were purchased from Aladdin Chemical Regent Company. Titanium mesh ( 60 meshes) was purchased from Shijiazhuang Hangxu Company. Analytical grade $\mathrm{NaNO}_{3}, \mathrm{KMnO}_{4}, \mathrm{NH}_{4} \mathrm{Cl}$ and $\mathrm{Na}_{2} \mathrm{SO}_{4}, 98 \%$ $\mathrm{H}_{2} \mathrm{SO}_{4}$, and $30 \% \mathrm{H}_{2} \mathrm{O}_{2}$ aqueous solution, were purchased from Shanghai Chemical Reagents Company, and they were used directly without further purification. Ultra-pure water (18 M $\Omega$ ) was produced by a Millipore System (Millipore Q, USA).

\section{Preparation of GO}

GO was prepared from graphite by a modified Hummers method. ${ }^{[11]}$ The obtained GO sediment was re-dispersed into water and treated by mild ultrasound for 30 min to access GO solution directly. Dried GO powder was obtained after freeze-drying of the sediment. 


\section{Preparation of latticed dried foam film of GO on Ti mash}

Aqueous solution of $\mathrm{GO}\left(5.0 \mathrm{mg} \mathrm{mL}^{-1}\right)$ was prepared by dispersing $\mathrm{GO}$ in ultra-pure water under mild ultrasound for 15 min, which was used for the GO latticed DFF preparation. Then a piece of titanium mash was evenly coated with $\mathrm{GO}$ to form latticed liquid foam films of aqueous solution of GO. The liquid films were then dried at $60^{\circ} \mathrm{C}$ to obtain a piece of latticed dried foam film of GO.

\section{Preparation of latticed rGO DFF films on Ti mash}

GO was reduced by a electrochemical method. In brief, electrochemical reduction of the as-formed GO DFF was performed on a $\mathrm{CHI660C}$ potentiostat-galvanostat $(\mathrm{CH}$ Instruments Inc.) at a voltage of $-1.0 \mathrm{~V}$ using $1 \mathrm{M} \mathrm{NH}_{4} \mathrm{Cl}$ as electrolyte.

\section{Preparation of polyaniline/rGO DFF films}

Electrochemical polymerization of polyaniline monomer onto the surface of rGO latticed DFFs was carried out by cyclic voltammetry at a scan rate of $40 \mathrm{mV} \mathrm{s}^{-1}$ and over a potential range of -0.2 to $1.2 \mathrm{~V}$ ( $v s$. SCE) with polyaniline (PANI) monomer in $1 \mathrm{M} \mathrm{H}_{2} \mathrm{SO}_{4}$ electrolyte.

\section{Supercapacitors and performance}

Electrochemical impedance spectroscopy (EIS) measurements and galvanostatic charge-discharge (GCD) experiments in a three-electrode electrochemical cell included a piece $\mathrm{Ti}$ mash containing active material (working electrode), a platinum wire (counter electrode), and an $\mathrm{Hg} / \mathrm{Hg}_{2} \mathrm{Cl}_{2}$ reference electrode. $\mathrm{CV}$ and EIS were carried out with $1 \mathrm{M} \mathrm{H}_{2} \mathrm{SO}_{4}$ as electrolyte. GCD and cycle GCD were carried out with $1 \mathrm{M} \mathrm{Na}_{2} \mathrm{SO}_{4}$ or $1 \mathrm{M}$ $\mathrm{H}_{2} \mathrm{SO}_{4}$ as electrolyte. For $\mathrm{CV}$ measurement, the specific capacitance of the PANI/rGO DFF was calculated using the equation $C_{\text {wt-sp }}=S / 2 m v U$, where $S$ is the integrated area of the CV curve, $m$ is the mass of PANI/rGO DFF material in the electrode, $v$ is the scan rate, and $U$ is the working potential window. For GCD, the specific capacitance of the PANI/rGO DFF was calculated using the equation $C_{\text {wt-sp }}=I \Delta t / m \Delta V$, where $l$ is the constant discharge current, $\Delta t$ is the discharging time, $m$ is the mass of PANI/rGO DFF material in the electrode, and $\Delta V$ is the voltage window. The volume specific capacitance was calculated using the equation $C_{\text {vol }}=C_{\text {wt-sp }} \times \rho{ }^{[8 a]}$

\section{Characterization}

The structures of materials were measured using a Sirion 200 FESEM at an accelerating voltage of $10 \mathrm{kV}$. X-ray photoelectron spectroscopy (XPS) was recorded on an Escalab MK II photoelectron spectrometer (VG Scientific, Ltd., United Kingdom).

\section{Results and Discussion}

Scheme 1 shows preparation process of latticed PANI/rGO dried foam films (DFF), which is typically a three-step process. At the first, nanosheets of GO were self-assembled to a series of latticed GO liquid foam films on the surface of Ti mesh, and then they were dried to form dried latticed GO DFFs. The size of GO nanosheets is about $2-5 \mu \mathrm{m}$, and the self-assembly of $\mathrm{GO}$ nanosheets in the liquid film driven by liquid crystalline phase formation of $2 \mathrm{D}$ sheets results in the formation of (a), (e) and density stacking film of GO in a frame of titanium mash. ${ }^{[9]}$ In the second step, the water-containing GO DFFs were reduced to $\mathrm{rGO}$ film through an electrochemical reduction process. At the end, PANI/rGO DFF films were prepared by an electrochemical polymerization of aniline on the surface of $\mathrm{rGO}$ DFF.

Figure 1 shows SEM images of as-prepared rGO DFF film and PANI/rGO composite materials of GO DFF film on Ti mesh,
Scheme 1 Preparation of the PANI/rGO DFF electrode by a typical three-step method

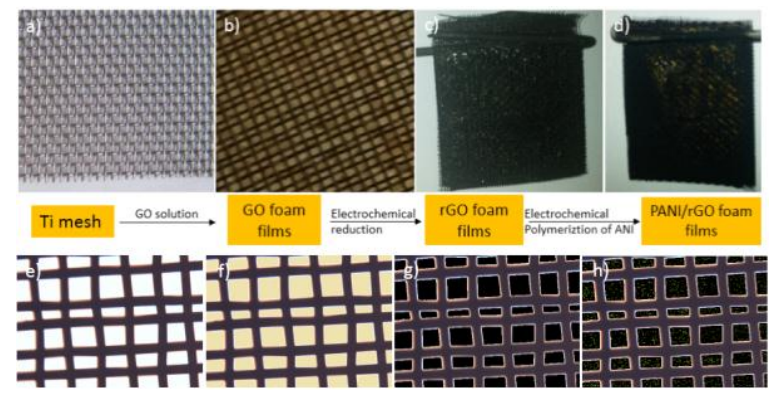

(a)-(d) Photoimages of clean Ti mesh, GO foam film on the mesh, rGO foam film, and PANI/rGO latticed DFFs on Ti mesh; (e)-(h) sketch maps of the relative products.

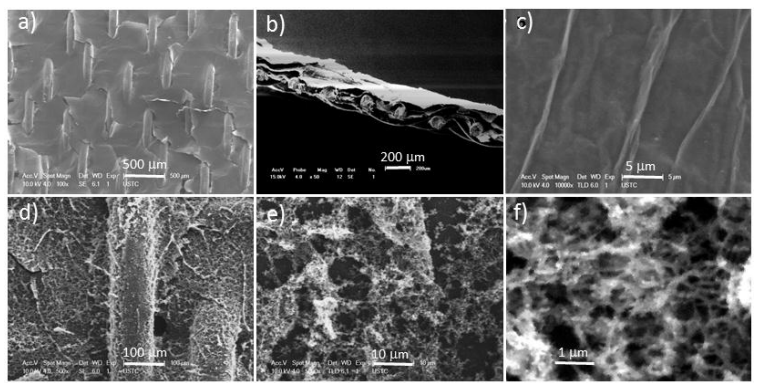

Figure 1 Photoimage (a) and SEM images (cross-section (b) and surface (c)) of as-prepared rGO DDF film on Ti mesh, and SEM images of the surface of PANI/rGO DFF film on Ti mesh at different magnifications ( $d, e$, and $f$ ).

and SEM images of the surface of the materials. Clearly, the $\mathrm{Ti}$ mesh was covered by an integrated rGO film, which was combined by plenty of latticed DFFs. The SEM cross-sectional image clearly showed that the film is thin and the thickness is about $500 \mathrm{~nm}$. After electropolymerization of aniline, the surface of rGO DFF film is covered by PANI layer as shown in Figure 1d. The zoom-in image of the surface clearly reveals that after in situ electropolymerization, PANI nanofibers with a diameter of about tens nanometers are tangled and twisted to form 3D porous networks on rGO DFF film. The specific surface areas of materials are measured with the adsorption of methylene blue, and the concentration change of methylene blue after the adsorption was measured by UV-Vis. The specific surface area of PANI/rGO composite is $1890 \mathrm{~cm}^{2} \mathrm{~g}^{-1}$, a relatively higher value, indicating that the composite is a potential active material for electrode of supercapacitors.

Electropolymerization of aniline on the rGO DFF film surface was achieved by applying potential cycling between -0.2 and $1.3 \mathrm{~V}$ at a scan rate of $40 \mathrm{mV} \mathrm{s}^{-1}$. Figure 2 shows the CV curves obtained during the polymerization up to 11 cycle. Clearly, the first redox process at about $0.22 \mathrm{~V}$ corresponds to electron transfer from/to electrodeposited polyaniline, and the second redox process at about $0.75 \mathrm{~V}$ probably corresponds to side reaction. The large positive current is due to electron transfer from PANI film corresponding to oxidation and electron transfer from aniline monomer to electrode corresponding to oxidation of aniline monomer for the precursor of PANI. All the results reveal the formation of PANI on the surface of rGO DFF film. ${ }^{[12]}$

XPS measurements could provide direct evidence of reduction of $\mathrm{GO}$ and deposition of polyaniline on the films. Figure 3 shows typical $\mathrm{C} 1 \mathrm{~s}$ and N1s XPS spectra of the as-prepared PANI/rGO DFF film. The curve was fitted as below: 


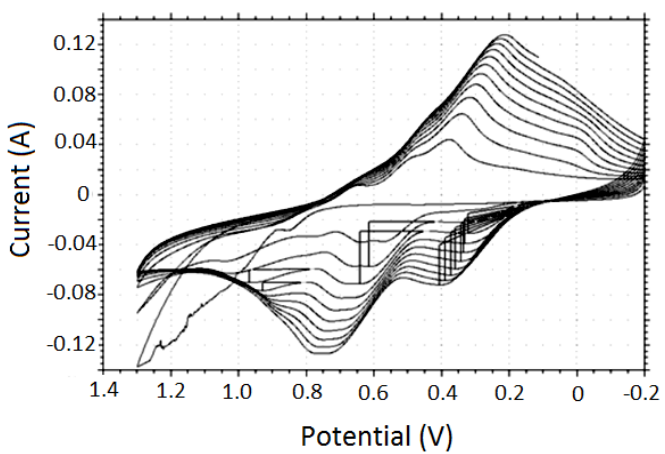

Figure 2 Cyclic voltammograms (CV) of the electropolymerization of aniline monomer on rGO DFF film in $\mathrm{H}_{2} \mathrm{SO}_{4}(1.0 \mathrm{M})$ solution.
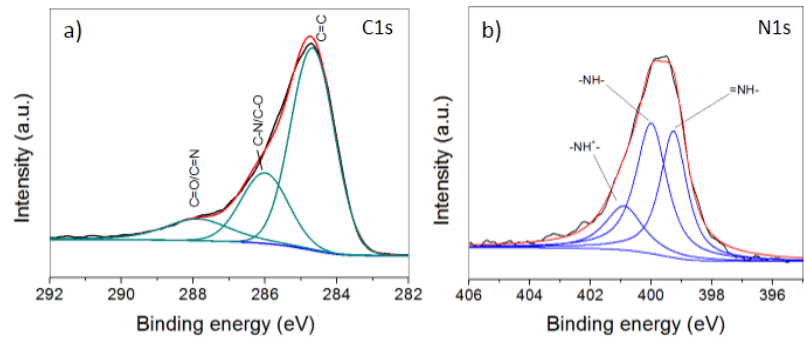

Figure 3 XPS C1s and N1s spectra of PANI/rGO film.

$\mathrm{C}=\mathrm{C}\left(\mathrm{sp}^{2}\right.$; peak 1; $\left.284.4 \mathrm{eV}\right), \mathrm{C}-\mathrm{N} / \mathrm{C}-\mathrm{O}$ (peak 2; $\left.286 \mathrm{eV}\right)$, $\mathrm{C}=\mathrm{O} / \mathrm{C}=\mathrm{N}$ (peak $3 ; 287.8 \mathrm{eV}$ ). ${ }^{[13]}$ Clearly, the typical peaks of both $\mathrm{rGO}$ and polyaniline can be assigned, and the elemental constant of $\mathrm{C}, \mathrm{N}$, and $\mathrm{O}$ are $68.42 \%, 11.3 \%$ and $19.6 \%$, respectively, indicating success preparation of target materials. The XPS N1s core-level spectrum of PANI/rGO film can be fit into three peaks as shown in Figure $3 \mathrm{~b}$. The major benzenoid-amine component $(\mathrm{NH})$ peak is observed at 400.0 $\mathrm{eV}$ along with quinoid-imine $(=\mathrm{NH})$ peak at $399.1 \mathrm{eV}$. The last peak at $400.09 \mathrm{eV}$ is attributed to positively charged nitrogen $\left(\mathrm{NH}^{+}\right)$, indicating that some nitrogen $(\mathrm{N})$ has been transmuted into protonated nitrogen species $\left(\mathrm{N}^{+}\right)$. The results reveal the formation of PANI on the surface of rGO DFF film, and the interaction between rGO DFF and PANI is mainly $\pi-\pi$ electron interactions.

The CV performance of the material is shown in Figure 4a. The CV curves are nearly rectangular in shape. They show two pairs of redox waves, which are indication of a typical pseudo-capacitive characteristic, attributed to redox transition of PANI between a semiconducting state (leucoemeraldine form) and a conducting state (polaronicemeraldine form), and the benzoquinone and aminoquinone couple. The calculated capacitance is $633.4,606.0$ and $479.1 \mathrm{~F} \mathrm{~g}^{-1}$ for 5,10 and 50 $\mathrm{mV} \mathrm{s}^{-1}$ scan rates, which is much higher that the pure rGO DFF film (149.6 $\mathrm{F} \mathrm{g}^{-1}$ at $\left.10 \mathrm{mv} \mathrm{s}^{-1}\right)$. The galvaostatic charge-discharge (GCD) method is usually to be utilized to evaluate the electrochemical capacitance of electrode, as shown in Figure 4b. The PANI/rGO DFF film shows the typical triangular-shape charge-discharge curves at various current densities, indicating that electrical double layer capacitances is the main source of the capacitance. However, the characteristic of the Faradaic capacitances of the PANI component can also be found. The symmetrical mirror-like curves indicates the reversible redox reaction also takes place in the electrode. The specific capacitance of the active materials can be calculated, and the $\mathrm{C}_{\mathrm{wt}}$ are $589.7,448.2$ and $413.1 \mathrm{~F} \mathrm{~g}^{-1}$ for $0.4,0.8$ and $1.6 \mathrm{~A} \mathrm{~g}^{-1}$ current densities (Figure 5).
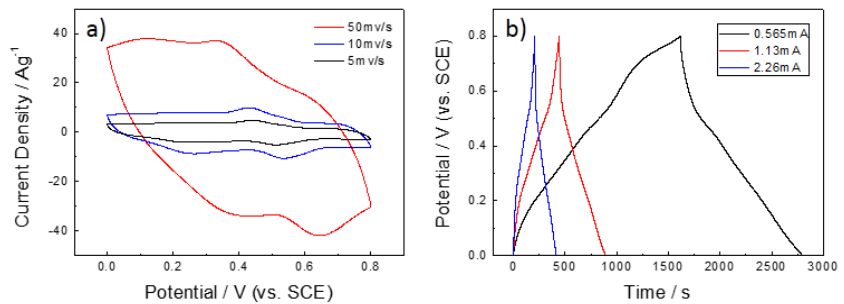

Figure 4 Electrochemical performances of PANI/rGO on $\mathrm{Ti}$ mesh measured in a three-electrode system. (a) Cyclic voltammograms at different scan rates of 5,10 , and $50 \mathrm{mV} / \mathrm{s}$; (b) Galvanostatic charge-discharge curves at different current densities.
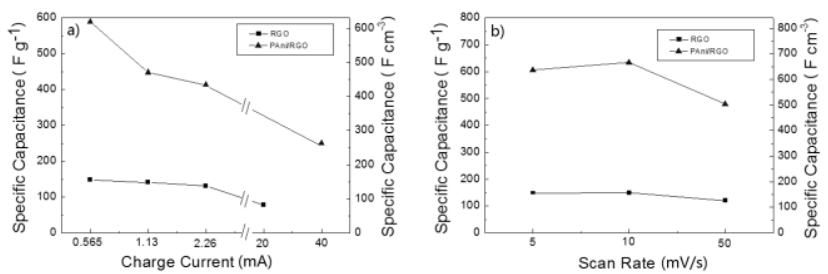

Figure 5 Relationship of specific capacitance of the PANI/rGO composite on the charged current (a) and scan rate (b).

To further evaluate cycle life of the samples, galvanostatic charge-discharge measurements are carried out at a current density of $10 \mathrm{~A} \mathrm{~g}^{-1}$ for 5000 cycles (Figure 6). The as fabricated $\mathrm{PANI} / \mathrm{rGO}$ material exhibits an excellent electrochemical stability with $75 \%$ of its initial capacitance. In addition, the active material retains about $45 \%$ of capacitance even in acidic electrolyte, indicating the PANI/rGO film has a widely potential application. Then, $\mathrm{N}$ content is $10.45 \%$ after 5000 cycles, indicating less $\mathrm{N}$ loss during the process.

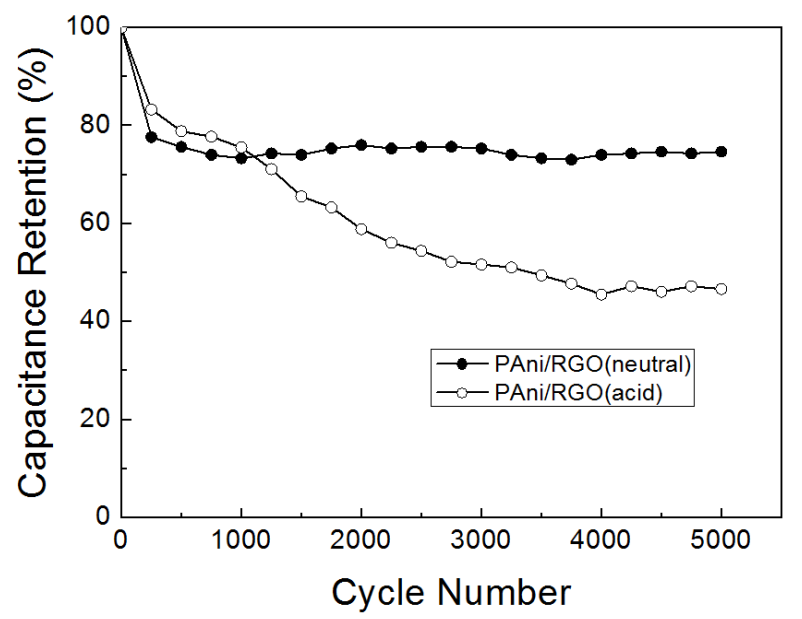

Figure 6 Cycle performance of the electrode in both acidic and neutral electrolytes.

Figure 7 shows Nyquist plot of $\mathrm{rGO}$ and PANI/rGO DFF films over the frequency range of $0.1 \mathrm{~Hz}$ to $10 \mathrm{kHz}$ by EIS. As expected, at a low frequency, their imaginary parts increased sharply and nearly vertical lines are observed, indicating their ideal capacitive behaviors. As a comparison of magnified data in the high-frequency range, for the PANI/rGO DFF film, a transition between $\mathrm{RC}$ semicircle and migration of electrolyte was observed at a frequency of about $966.8 \mathrm{~Hz}$, corresponding to a smaller resistance of $0.25 \mathrm{ohms}$ than $0.35 \mathrm{ohms}$ at 312.5 $\mathrm{Hz}$ of the rGO DFF. 


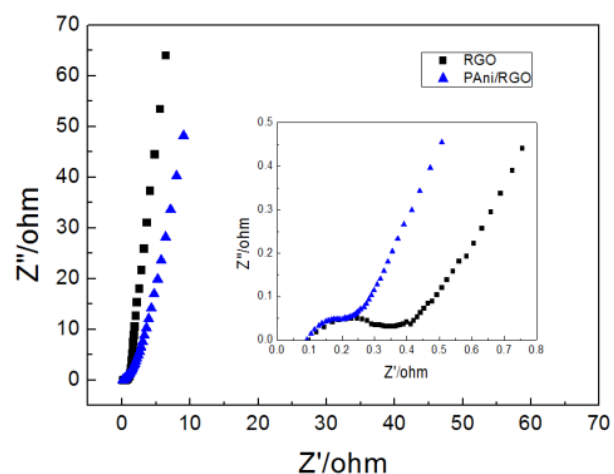

Figure 7 Niquist plot of the $\mathrm{rGO}$ and PANI/rGO DFF films on $\mathrm{Ti}$ mesh and magnified diagram in the high frequency region (inset).

\section{Conclusions}

Free-standing film of rGO has been prepared by a dried foam film technique following an electrochemical reduction on the surface of $\mathrm{Ti}$ mesh, which also works as the collector of electrode. Then, pyrrole was electrochemical polymerization on the surface of rGO film, and polyaniline/rGO latticed foam films were obtained at the end. The materials can directly work as the electrode for supercapacitors without addition of binder or additives. The performance of supercapacitor is high and stable, and it provides a new method to prepare graphene based active materials for supercapacitors.

\section{Acknowledgement}

This work is supported by the National Natural Science Foundation of China (Nos. 51873201 and 51673180) and Shaanxi YanChang Petroleum (Group) Co., Ltd.

\section{Author Contributions}

W. C. and J. C. carried the experiment, W. C. wrote this paper, and L. Y. designed the research.

\section{Conflict of Interest}

The authors declare no conflict of interest.

Copyright ( 2021 Wufeng Chen, Junhao Chen, Lifeng Yan. This article is an open access article distributed under the terms and conditions of the Creative Commons Attribution (CC BY) license (http://creativecommons.org/licenses/by/4.0/). The use, distribution or reproduction in other forums is permitted, provided the original author(s) or licensor are credited and that the original publication in this journal is cited, in accordance with accepted academic practice. No use, distribution or reproduction is permitted which does not comply with these terms.

\section{References}

[1] Han, S.; Wu, D. Q.; Li, S.; Zhang, F.; Feng, X. L. Porous Graphene Materials for Advanced Electrochemical Energy Storage and Conversion Devices. Adv. Mater. 2014, 26, 849-864.

[2] (a)Simon, P.; Gogotsi, Y. Capacitive Energy Storage in Nanostructured Carbon-Electrolyte Systems. Acc. Chem. Res. 2012, 46, 1094-1103; (b) Xing, T.; Ouyang, Y.; Chen, Y.; Zheng, L.; Wu, C.; Wang, X. P-doped ternary transition metal oxide as electrode material of asymmetric supercapacitor. J. Energy Storage 2020, 28, 101248; (c) Zheng, L.; Xing, T.; Ouyang, Y.; Wang, Y.;

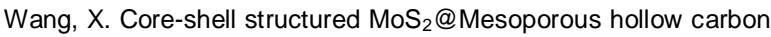
spheres nanocomposite for supercapacitors applications with enhanced capacitance and energy density. Electrochim. Acta 2019,
298, 630-639

[3] Novoselov, K. S.; Geim, A. K.; Morozov, S. V.; Jiang, D.; Zhang, Y.; Dubonos, S. V.; Grigorieva, I. V.; Firsov, A. A. Electric field effect in atomically thin carbon films. Science 2004, 306, 666-669.

[4] Yang, X. W.; Cheng, C.; Wang, Y. F.; Qiu, L.; Li, D. Liquid-Mediated Dense Integration of Graphene Materials for Compact Capacitive Energy Storage. Science 2013, 341, 534-537.

[5] (a) de Oliveira, H. P.; Sydlik, S. A.; Swager, T. M. Supercapacitors from Free-Standing Polypyrrole/Graphene Nanocomposites. J. Phys. Chem. C 2013, 117, 10270-10276; (b) Wang, S.; Ma, L.; Gan, M.; Fu, S.; Dai, W.; Zhou, T.; Sun, X.; Wang, H.; Wang, H. Free-standing 3D graphene/polyaniline composite film electrodes for high-performance supercapacitors. J. Power Sources 2015, 299, 347-355.

[6] (a) Liu, K.; Chen, Y.-M.; Policastro, G. M.; Becker, M. L.; Zhu, Y. Three-Dimensional Bicontinuous Graphene Monolith from Polymer Templates. ACS Nano 2015, 9, 6041-6049; (b) Wu, Z.-S.; Sun, Y.; Tan, Y.-Z.; Yang, S.; Feng, X.; Muellen, K. Three-Dimensional Graphene-Based Macro- and Mesoporous Frameworks for High-Performance Electrochemical Capacitive Energy Storage. J. Am. Chem. Soc. 2012, 134, 19532-19535.

[7] (a) Zou, J.; Kim, F. Diffusion driven layer-by-layer assembly of graphene oxide nanosheets into porous three-dimensional macrostructures. Nat. Commun. 2014, 5; (b) Xu, D.; Xu, Q.; Wang, K.; Chen, J.; Chen, Z. Fabrication of Free-Standing Hierarchical Carbon Nanofiber/Graphene Oxide/Polyaniline Films for Supercapacitors. ACS Appl. Mater. Interfaces 2014, 6, 200-209; (c) Qin, K.; Kang, J.; Li, J.; Shi, C.; Li, Y.; Qiao, Z.; Zhao, N. FreeStanding Porous Carbon Nanofiber/Ultrathin Graphite Hybrid for Flexible Solid-State Supercapacitors. ACS Nano 2015, 9, 481-487; (d) Ji, J.; Li, Y.; Peng, W.; Zhang, G.; Zhang, F.; Fan, X. Advanced Graphene-Based Binder-Free Electrodes for High-Performance Energy Storage. Adv. Mater. 2015, 27, 5264-5279; (e) Khosrozadeh, A.; Xing, M.; Wang, Q. A high-capacitance solidstate supercapacitor based on free-standing film of polyaniline and carbon particles. Appl. Energy 2015, 153, 87-93.

[8] (a) Feng, X.; Chen, W.; Yan, L. Reduced graphene oxide hydrogel film with a continuous ion transport network for supercapacitors. Nanoscale 2015, 7, 3712-3718; (b) Feng, X.; Chen, W.; Yan, L. Electrochemical reduction of bulk graphene oxide materials. RSC Adv. 2016, 6, 80106-80113.

[9] Chen, W.; Yan, L. Centimeter-Sized Dried Foam Films of Graphene: Preparation, Mechanical and Electronic Properties. Adv. Mater. 2012, 24, 6229-6233.

[10] (a) Lu, X.; Yu, M.; Wang, G.; Tong, Y.; Li, Y. Flexible solid-state supercapacitors: design, fabrication and applications. Energy Environ. Sci. 2014, 7, 2160-2181; (b) Dallas, P.; Georgakilas, V. Interfacial polymerization of conductive polymers: Generation of polymeric nanostructures in a 2-D space. Adv. Colloid Interface Sci. 2015, 224, 46-61.

[11] (a) Becerril, H. A.; Mao, J.; Liu, Z.; Stoltenberg, R. M.; Bao, Z.; Chen, Y. Evaluation of Solution-Processed Reduced Graphene Oxide Films as Transparent Conductors. ACS Nano 2008, 2, 463-470; (b) Hummers, W. S.; Offeman, R. E. Preparation of Graphitic Oxide. J. Am. Chem. Soc. 1958, 80, 1339.

[12] Baba, A.; Tian, S.; Stefani, F.; Xia, C.; Wang, Z.; Advincula, R. C.; Johannsmann, D.; Knoll, W. Electropolymerization and doping/dedoping properties of polyaniline thin films as studied by electrochemical-surface plasmon spectroscopy and by the quartz crystal microbalance. J. Electroanal. Chem. 2004, 562, 95-103.

[13] Chen, W. F.; Yan, L. F.; Bangal, P. R. Preparation of graphene by the rapid and mild thermal reduction of graphene oxide induced by microwaves. Carbon 2010, 48, 1146-S1152.

Received August 18, 2020 Accepted October 17, 2020 